\title{
Design of the Composite Steel-Glass Beams with Semi-Rigid Polymer Adhesive Joint
}

\author{
Michal Netusil and Martina Eliasova \\ Department of Steel and Timber Structures, Faculty of Civil Engineering, Czech Technical University in Prague, Prague 140 00, Czech \\ Republic
}

\begin{abstract}
New and high transparent structural element, steel-glass composite beam was developed in respect to fabrication, static-structural and architectural criteria and consists of steel flanges and glass web assembled together by semi-rigid polymer adhesive, which is the key element of whole composite system. These beams can be used mainly as members of high transparent roof or floor structure as well as stiffening fins for large area glass facades. This paper deals with experimental research performed at CTU (Czech Technical University) Prague, which started by adhesive selection and initial material tests by ISO527, continued via small-scale steel-glass connection tests and graduated by full-scale tests of hybrid beams with the span of 4 m. Generalized results of these experiments, analytical and numerical studies serve as device, how to accurately predict the behavior of the beam, describe the stress distribution along the cross section and safely and economically design such a kind of structure with semi-rigid shear connection, made by polymer adhesive.
\end{abstract}

Key words: Steel-glass composite, beam, polymer, adhesive, FE modeling, semi-rigid joint.

\section{Introduction}

Due to the intensive progress and research on the field of glass structures, possibilities for using glass as a load carrying elements are increasing every day. Different types of hybrid constructions, consisted of glass and another material (timber, concrete, laminated reinforcement, etc.) are analyzed or even newly developed focusing on an optimal structural interaction between both materials and in respect to architectural, static-structural and fabrication criteria. Current research work can be found in Refs. [1-4]. Main goal of composite (stiffened) glass structures is to increase the load bearing capacity, stiffness and robustness (safety) in comparison with pure glass structures, which are brittle and always fail suddenly and without previous warning. New, hybrid steel-glass beam consists of steel flanges and glass web bonded

Corresponding author: Michal Netusil, $\mathrm{PhD}$, research fields: steel and timber structures design, composite steel-concrete structures, glass structures, stiffened glass elements, adhesive bonding technology and experimental research. E-mail: michal.netusil@fsv.cvut.cz. together, see Fig. 1. Long glued joint, performed by semi-rigid polymer adhesive is the key element of whole composite structure and therefore special emphasis has to be dedicated to the detailing of the steel-glass interface and choice of the suitable adhesive. Bonded hybrid structures are favorable where each material is used in an optimized way according to its mechanical properties. This can only be achieved by assigning the adhesive a real load-bearing role.

\section{Performance in Use of Composite Beams}

Hybrid floor girders (or façade elements) were developed, where flanges of steel and webs of glass are assembled to I-beams using adhesive connections. This allows a smooth load introduction into the glass panes. The shear force is carried by the glass web, whereas the bending capacity of the hybrid beam is significantly increased by slender steel flanges compared to the pure glass pane. The shear forces between steel and glass are only sustained by the adhesive between them. Different details of 
connection between steel and glass, designed to be used in hybrid beams are drawn in Fig. 2.

To maximize the exploitation of steel and glass, the adhesive has to ensure an adequate stiffness to provide optimal structural interaction between both materials, but on the other hand must be flexible enough to compensate different temperature elongation of steel and glass allowing a reduction resp. redistribution of stress peaks or other constraints. In general, glued joint offers a bigger ability to assemble thinner materials or to connect different materials together as well as aesthetic appearance and reduction of self-weight. Adhesive layer itself also provides a protection from direct contact between steel and glass, which always has to be avoided in glass structure engineering. However the load-bearing capacity of such beams is governed, apart from the mechanical and strength characteristics of the adherent, by ageing, temperature and creeping. Therefore the choice of adhesives has a central role in the research because the

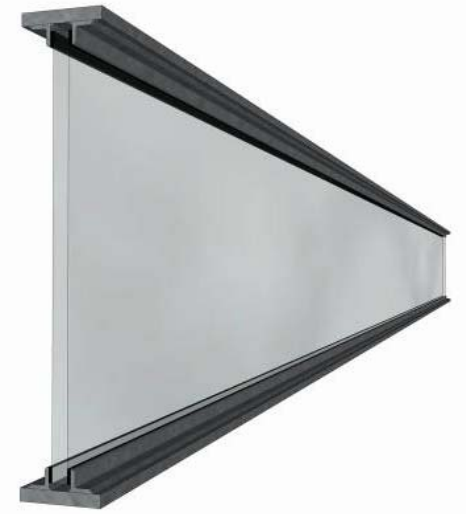

Fig. 1 Steel-Glass composite beam-view.
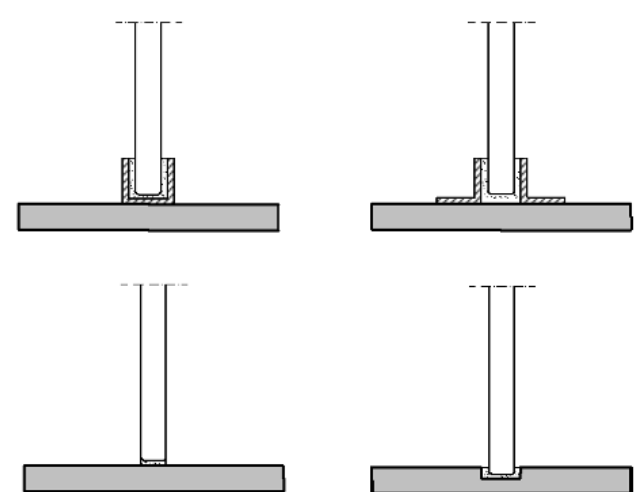

Fig. 2 Details of bonded connection between web and flanges. load carrying-capacity of the composite section depends primarily on mechanical material characteristics of the joint. Furthermore to ensure a traceable quality and workmanship of the adhesive connection the production process beginning with the storage of the components, going on to the surface pre-treatment to the point of manufacturing and post processing of the bonded joint has to be defined and controlled clearly. Hybrid steel-glass beams are composite structural elements comprising steel flanges and glass webs, with adhesive bonded joints between the steel and glass. Combining these materials in any building element permits the structural engineer to utilize the strength and ductility of the steel, while allowing the architect to benefit from the aesthetic beauty of the glass. Glass and steel together create a light and airy appearance, minimizing the obtrusiveness of the structure and maximizing the natural daylight available within the building. Natural daylight is known to improve the health and productivity of building occupants and reduces the demand for electric lighting (therefore reducing carbon emissions). Glass and steel together give the impression of a modern, clean environment.

\section{Selection of Adhesives}

Requirements on adhesive layer are mainly focused on strength and stiffness. Common adhesives can be divided according to their modulus of elasticity and shear modulus into flexible-elastic (i.e., silicones, modified silicones and polyurethanes) and rigid (i.e., epoxy resins, acrylics). Stiff adhesives offer extremely high strength but very low elongation in comparison with elastic adhesives, which show elongation at break even more than 250\%. Practical application of different types of polymer adhesives depends on their behaviour under loading. During the selection, special emphasis has to be devoted to the UV (ultraviolet) stability and long-time behaviour of chosen adhesives. UV unstable adhesives, like most of the polyurethanes, have to be protected from UV lights by using special 
primer coating also on the side of the glass pane, because there is a risk of UV lights propagation also by the reflection inside the glass pane.

Definitely, two-component adhesives or adhesives with booster system should be used for such a kind of connection, where the width of the connection is too large for humidity curing (over $30 \mathrm{~mm}$ ). From previous research came out, that one-component adhesives (mainly polyurethanes), which are cured by air humidity, cannot harden for depths wider than approx. $15 \mathrm{~mm}$ in a reasonable period of time. The booster component provides uniform hardening of the adhesive layer, process of curing doesn't depend on air humidity and the whole curing is finished in hours and not in days as for one component adhesives. Another important task of the connection design is to find an optimal adhesive thickness, which fulfils the requirements on stiffness and load carrying capacity, provides sufficient elongation (or shear strain) and also compensates possible geometrical imperfections and balances tolerances of the connected surfaces during the fabrication of the beam. All adhesives should be also chosen regarding to their open time and pot-time, which is very important in respect to fabrication criteria. Some of adhesives can be applied by gap-filling, but other more viscous ones have to be compressed by the components that have to be connected. Both kinds of adhesive application methods for beam fabrication are applicable for the production of the hybrid beams with the total length up to $6 \mathrm{~m}$ (usual maximal construction length of one glass pane).

The final choice of adhesive is additionally influenced by arising temperature elongations as well as susceptibility for ageing and creeping. Ageing is a process that strongly depends on the adhesive system. Ageing, corrosion and temperature changes occur under natural atmospheric exposure. According to the climatic zone these effects are more or less pronounced and can lead to chemical and molecular changes in the adhesives structure. Commonly affected are the boundary layer and the adhesion between adhesive and substrate surfaces, but there is also a considerable influence to the cohesion of the adhesive itself. Besides a reduction of the adhesion and a tendency for adhesive interface failure because of peeling stresses or stress peaks, ageing effects go along with embrittlement and a decrease of strength.

Concerning the choice of adhesives for experimental research, major aim was the investigation of adhesives systems with higher stiffness and strength as provided by standard silicones. Silicones are generally used in structural glazing facades, but offer here only little structural features. With reference to previously mentioned requirements on suitable bonding material, wide range of adhesives with different mechanical and deformational properties was involved to the experimental program. Final choice of adhesives was supported by SIKA CZ and includes almost all common used material types of polymer adhesives. Set of adhesives starts with a very stiff epoxy resin, goes down via acrylics and polyurethanes to very flexible silicone, which is included only as a border element to bounder the set of adhesive behaviour from below

\section{Investigation of Adhesive Behavior}

First of all, material tests according to EN ISO527 (Plastics, determination of tensile properties), see Fig. 3, were performed for all chosen adhesives (epoxy resin, acrylic, polyurethane and silicone). Real material properties (progress of tensile stiffness, elongation at break and whole stress-strain diagrams) were determined for increasing uni-axial load. These

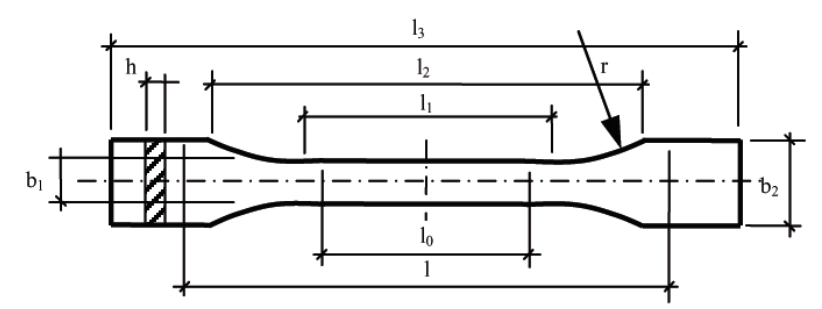

Fig. 3 Standardized shape of test specimen by ISO527. 
initial tests gave an idea about the important mechanical properties and suitability of chosen adhesives for key element of hybrid steel-glass beam.

Each adhesive shows different behaviour under increasing load regarding to the thickness of the joint. Most important for hybrid beam design is the behaviour of adhesive layer in shear. Therefore, small scale steel-glass connection tests were performed, see Fig. 4.

By increasing thickness of the joint over the ideal value, possible elongation (or slope) of the adhesive layer increases too, but the connection loses its load carrying capacity. This was proved experimentally for common types of adhesives by steel-glass connection tests, see Fig. 5, where representative curves of the shear stress - strain diagrams are drawn for each of the tested adhesives (except stiff epoxy resin) and influence of the thickness of the joint is significant especially for acrylic and polyurethane too [5]. One of the main tasks of the connection design is to find the state of the adhesive thickness, where the glued joint can fulfill the requirements on load carrying capacity and also provides a necessary temperature elongation (or shear slope) and compensation of possible geometrical imperfections of connected surfaces during fabrication of the beam.

Finite-element method was chosen as a device for numerical studies. Volume models of tested glued connections were created by software package Ansys 11, see Fig. 6. Steel and glass parts were modeled by a member SOLID45. This element type can be used also for adhesive, but only in case of stiff connection with small deformations and behaviour of adhesive close to linear elastic. Other element types, i.e., SOLID185, have to be used in case of compliant connection with significant non-linear behaviour and huge deformation in hundreds of percent.

Models of the adhesive layer were developed and calibrated for each tested adhesive to be in conformity with experimental results. Adhesives, which are tougher, for example epoxy resins or acrylics could

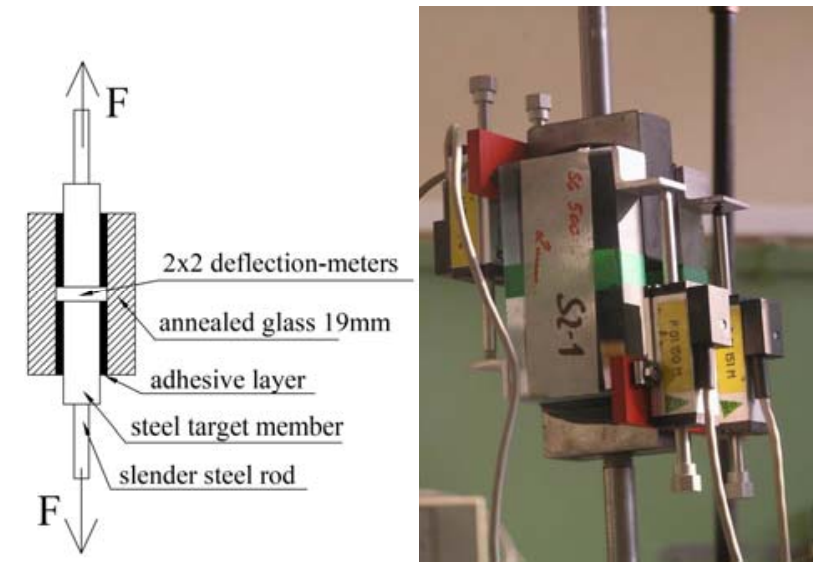

Fig. 4 Setup of the steel-glass connection tests.

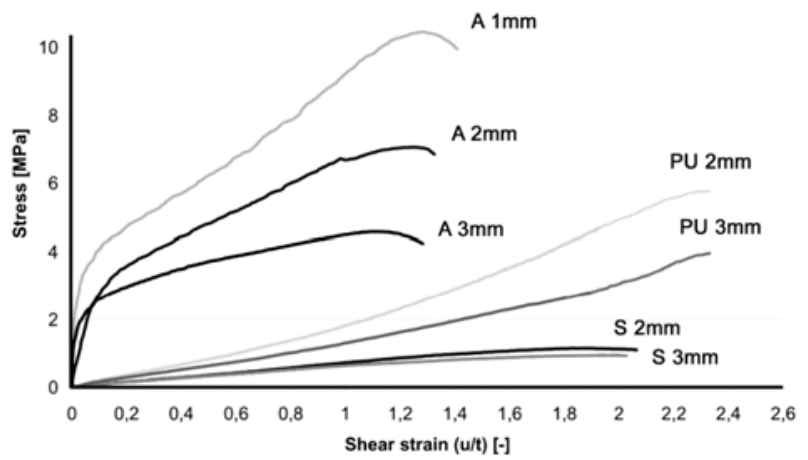

Fig. 5 Shear stress-strain diagrams of different adhesives in connection (S-silicone, A-acrylic, PU-Polyurethane booster system) [5].

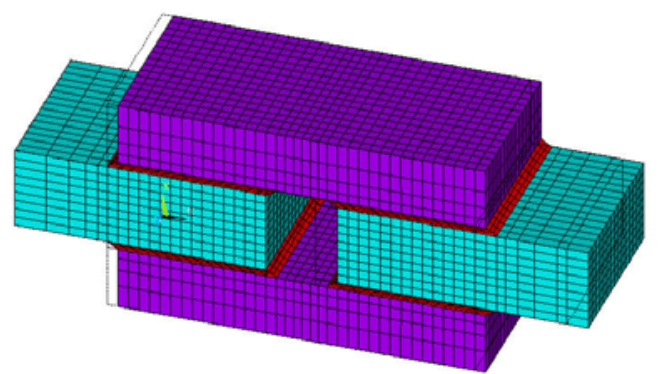

Fig. 6 FE model of the steel-glass connection tests.

have been modeled as multi-linear elastic (exceptionally as a linear elastic in case of very stiff epoxies), with using appropriate input material parameters. There is a possibility to use phenomenological material models, which are included in most of the common used finite element software. Hyper-elastic models, i.e., Mooney-Rivlin, Arruda-Boyce or Ogden, can very accurate predict the behaviour of compliant adhesives like silicones or tested polyurethanes, see example in Fig. 7. 


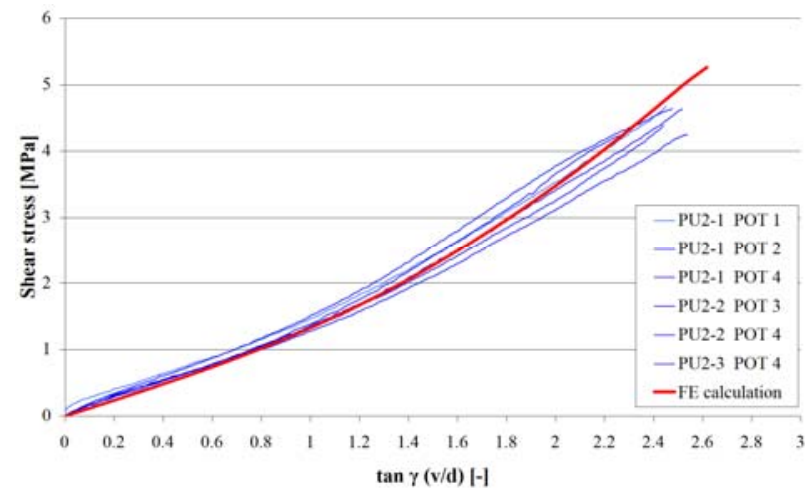

Fig. 7 Calibrated FE model of polyurethane in connection.

Their calibration isn't easy because a lot of input parameters are needed and longer calculation time is required. Whole process of calibration of the material model is more complex, but these models can withstand extensive deformations.

\section{General Analytic Model of the Hybrid Beam}

The hybrid steel glass beam is a flexible composite section element. Load bearing characteristics significantly depend on the stiffness of the connection between flanges and web. This is presented in Fig. 8. First limit case is Beam 1, which presents a rigidly connected section. There is no displacement between steel and glass. Steel flanges are loaded with moments and high normal forces. This leads to a high load carrying capacity. Second limit case is Beam 3 which consist of unconnected section members. There is a high displacement between steel and glass. All section members are loaded with bending moments only. This beam has low carrying capacity and high deflections compared to Beam 1. The behaviour of the bonded hybrid steel-glass beam is located in between these two cases as a Beam 2. There is a displacement between steel and glass due to adhesive flexibility. The load carrying capacity generally increases with the increase of the connection stiffness, because of the higher normal forces in the flanges.

Shear resistance and shear modulus of adhesive are important characteristics and have high influence on the load carrying capacity and structural behaviour of

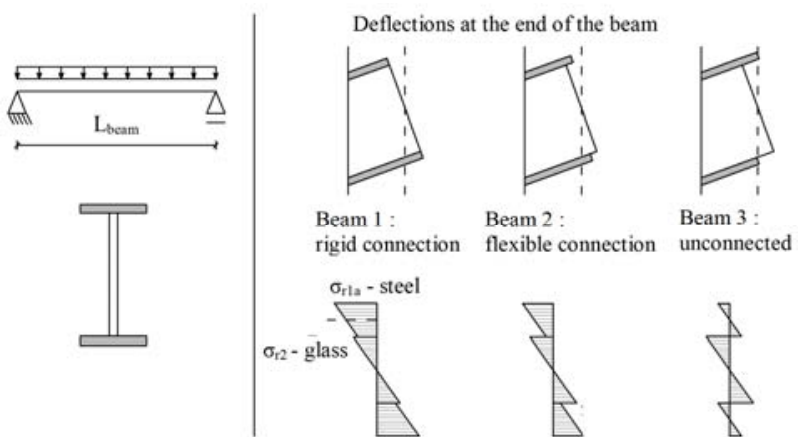

Fig. 8 Load bearing scheme of flexible composite section.

the hybrid steel glass beam. The stiffness of the bonded connection depends on shear stiffness of the adhesive (shear modulus $\mathrm{G}$ ) and on the geometry of the joint. Increasing thickness of the adhesive will decrease the stiffness of the joint. Enlargement of the width of joint leads to an enlargement of stiffness of whole connection, therefore direct connection on the face of the glass-sheets have a low stiffness because of the small bonding area. Other geometries offer larger bonded surface and therefore higher effective connection stiffness.

Flexible composed hybrid-beams can be pre-designed according to Möhler [6], who developed his method (which is today known as the $\gamma$-method) in 1956 for the design of timber structures. It can be applied to the hybrid steel glass beam considering the different material properties of steel and glass as well as the flexibility of the joint with an effective moment of inertia with reduced contribution of the flanges. Therefore it is qualified very well for the pre-design of hybrid steel glass beams and was applied by author to solved problem as following.

Assume the simplest bonded connection between steel flange and glass web - direct connection on the face of glass-sheet with one adhesive layer, see Fig. 9.

Average stiffness of the connection $\mathrm{K}_{\mathrm{K}}$ (along its length) can be determined by Eq. (1), where $G_{K}$ is experimental found shear modulus of used adhesive (MPa), $b$ is the width of adhesive (mm) and $t_{k}$ is the thickness of the adhesive layer (mm).

$$
K_{K}=G_{K} \cdot \frac{b}{t_{k}}
$$




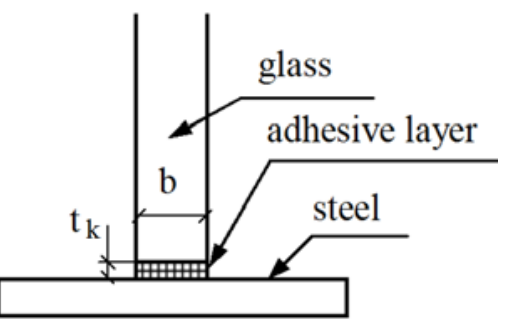

Fig. 9 The simplest connection between web and flanges.

Efficiency factor $\gamma$ and effective moment of inertia $I_{Y, \text { eff }}$ of double symmetric cross section are given by the following formulas:

$$
\begin{gathered}
\gamma=\frac{1}{1+k} \\
I_{y, \text { eff }}=2 \cdot I_{a}+n \cdot I_{G}+2 \cdot \gamma \cdot A_{a} \cdot z_{a}^{2}
\end{gathered}
$$

where

$$
\begin{gathered}
k=\pi^{2} \cdot \frac{E_{a} \cdot A_{a}}{L^{2} \cdot K_{K}}, \\
n=\frac{E_{G}}{E_{a}},
\end{gathered}
$$

where $E_{a}$ is the Young's modulus of steel (MPa), $E_{G}$ is the Young's modulus of glass (MPa), $A_{a}$ is the area of one steel flange $\left(\mathrm{mm}^{2}\right), L$ is the span of the beam $(\mathrm{mm}), I_{a}$ is the moment of inertia of one steel flange $\left(\mathrm{mm}^{4}\right), I_{G}$ is the moment of inertia of the glass web $\left(\mathrm{mm}^{4}\right), z_{a}$ is the vertical distance between the centre of gravity of the steel flange and centre of gravity of whole cross section (mm).

Values of the normal stress distribution along the cross section, see Fig. 10, can be obtained from following formulas:

$$
\begin{gathered}
\sigma_{a}= \pm \frac{M_{y}}{I_{y, e f f}} \cdot\left(\gamma \cdot z_{a}+\frac{t_{f}}{2}\right) \\
\sigma_{g}= \pm \frac{M_{y}}{I_{y, e f f}} \cdot \frac{h_{w}}{2} \cdot n
\end{gathered}
$$

where, except the values described above, $M_{y}$ is the maximal bending moment caused by external load $(\mathrm{N} \cdot \mathrm{mm}), t_{f}$ is the thickness of the steel flange $(\mathrm{mm})$ and $h_{w}$ is the height of the glass web (mm).

Shear stress within the adhesive layer can be calculated (simplified) by Eq. (8), where $Q$ is the maximum shearing force caused by external load.

$$
\tau=\frac{Q \cdot \gamma \cdot z_{a} \cdot A_{a}}{I_{y, \text { eff }} \cdot b}
$$

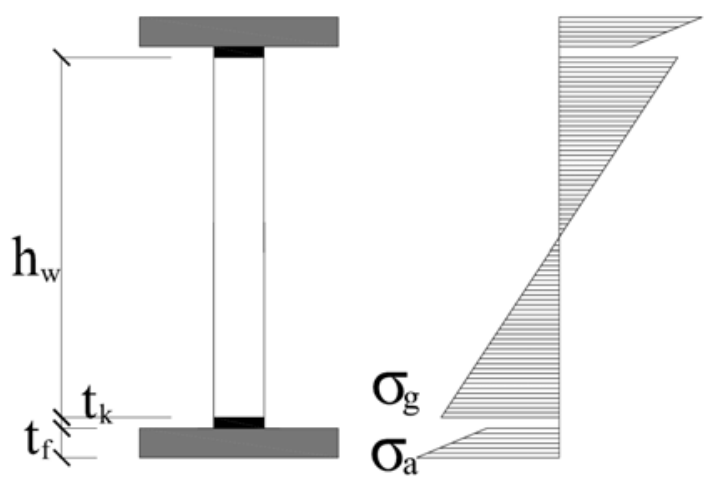

Fig. 10 Normal stress distribution along the cross section.

Finally, vertical deflection in the middle of the span of the hybrid beam subjected to uniformly distributed load $q(\mathrm{kN} / \mathrm{m})$ can be determined by Eq. (9):

$$
\delta=\frac{5}{384} \cdot \frac{q \cdot L^{4}}{E_{a} \cdot I_{y, \text { eff }}} .
$$

\section{Full-Scale Tests}

Hybrid beams with the total length of $4.25 \mathrm{~m}$ (4 m span) was subjected to 4-point bending tests until the glass failure, see Fig. 11, where the lateral support in the middle of the span was arranged to avoid lateral-torsion buckling effects. Beams were consisted of steel grade S235 flanges, dimensions $100 \times 10 \mathrm{~mm}$ and glass web composed of double-layered toughened glass $2 \times 12 \mathrm{~mm}$ with the height of $290 \mathrm{~mm}$. Glass web was installed into polyamide bedding placed in additional $U$ profile, welded continuously on the flanges. Polyamide bedding doesn't contribute to structural behaviour (load bearing capacity), but avoid pressure contact of glass edge and steel flange as well as vertical shear in adhesive layer near supports and load introduction points. Bonded joint was performed only on the sides of the glass pane by gap filling, see detail of described connection in Fig. 12.

A few beams were created with the direct connection between steel flanges and glass web (without any helpful profile) and $19 \mathrm{~mm}$ thick single-layered toughened glass was used, see Fig. 13. In this case, glued joint was performed on the edge of the glass web, it means, the area of adhesion is restricted by the thickness of the glass web. This set-up was chosen only 


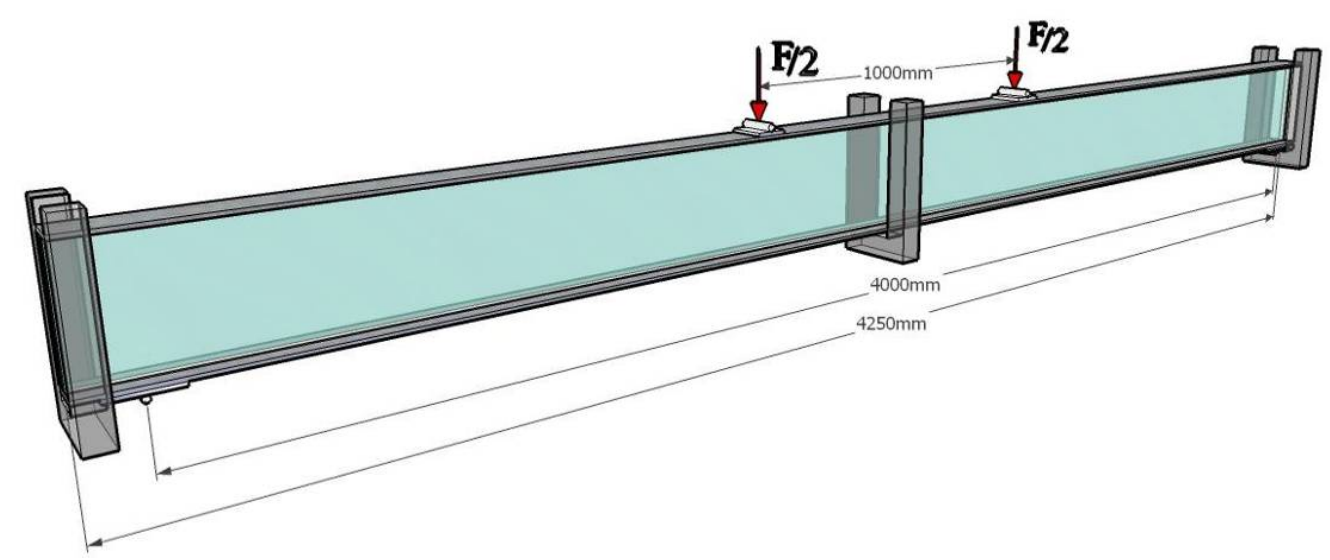

Fig. 11 Set-up of the full-scale tests of hybrid beams.

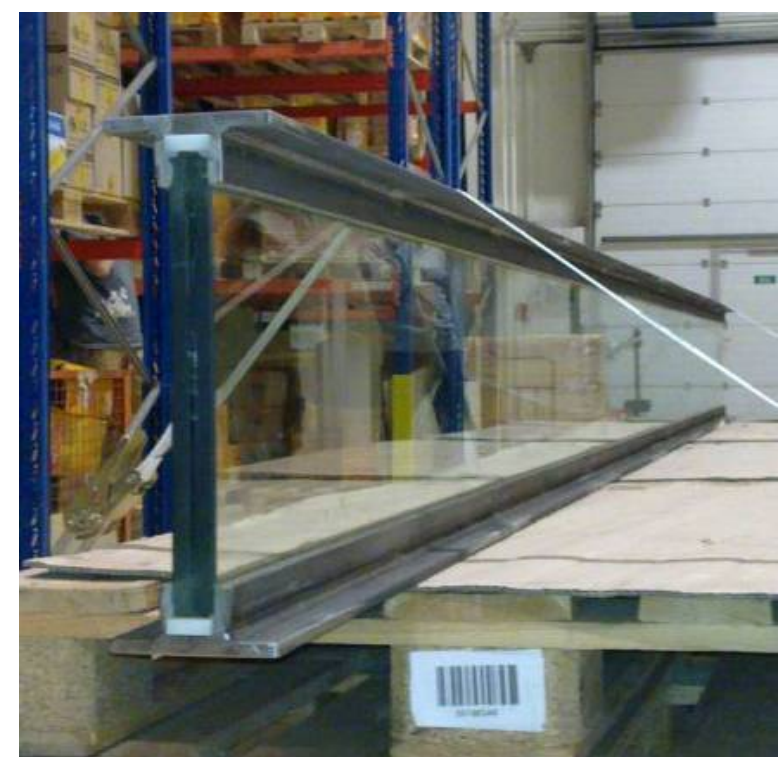

Fig. 12 Hybrid beam with U-profile connection.

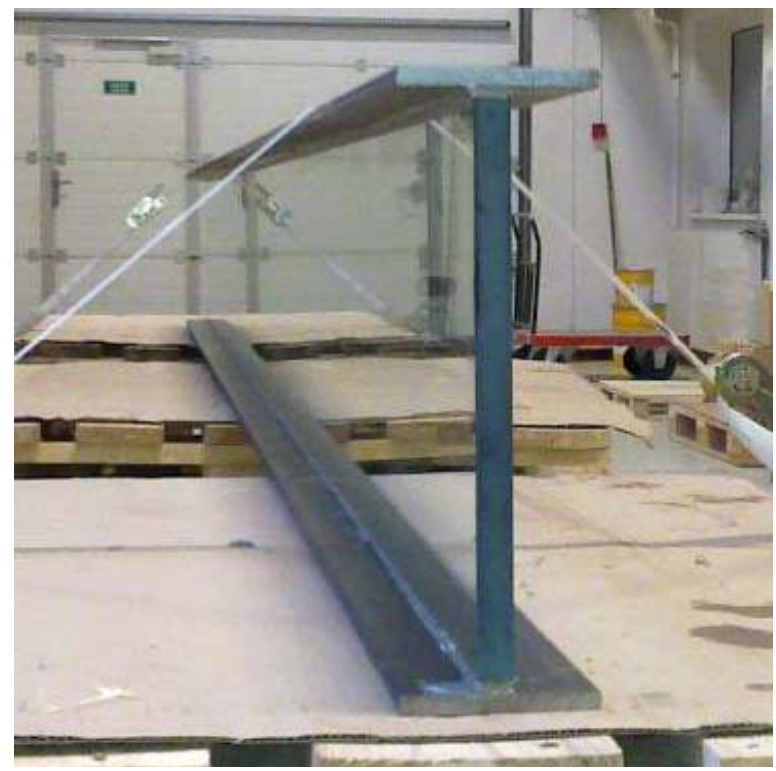

Fig. 13 Hybrid beam with direct connection. because of simplicity, to minimize other effects of PVB foil and side connection, to evaluate hand calculation methods and verify numerical models. Single-layered glass will never be used in real structure because of neglected residual load bearing capacity and sudden collapse without any warning before.

Final choice of adhesive for hybrid beams resulted to two component acrylic adhesive and polyurethane booster system. Very stiff epoxy resin was taken of from experimental program because of neglected possible elongation (shear slope), which is required because of temperature changes and redistribution of stress peaks (near support and load introduction points) in contact of the adhesive and glass surface. Compliant silicone was taken off as well because of very low stiffness and strength.

Hybrid beams with direct connection between flanges and web are more attractive in terms of architectural design, but they have lower load bearing capacity in comparison with the U-profile connection, where also the residual capacity is higher.

Measured values were deflection in the middle of the span and under the load introduction points, normal stresses along the length of the beam and stress distribution in hybrid cross-section in different sections and horizontal sliding between steel and glass in the end section. Measured normal stress near the edge of the glass web was close to $120 \mathrm{MPa}$ before failure in all of the tested specimens. Ultimate load varies by chosen 
adhesive and detail of connection. Final choice of adhesive is influenced by the requirements of temperature changes and future type of load acting on the structure. In case of direct connection and stiff acrylic adhesive, ultimate total force in the middle of the span reached average value of $F=75 \mathrm{kN}$, which was very successful if we take on mind that load bearing capacity of pure glass fin subjected to bending, caused by the force in the same set-up, would be about $42 \mathrm{kN}$. It means improvement of the load bearing capacity for $78 \%$ by the minimum of steel and simple direct connection. By the use of polyurethane in the same contact, $61 \mathrm{kN}$ was achieved as average ultimate load. PU adhesive has advantage in the elongation, see Fig. 5, and can be used even to the environment with high temperature changes and more dynamic load, but the load carrying capacity decreases because of lower rigidity of horizontal shear connection. Much higher ultimate load can be applied on the beams with U-profile connection, $F=130 \mathrm{kN}$ for acrylic adhesive and $F=105 \mathrm{kN}$ for polyurethane. Safely designed beam has to carry at least service load when one pane of laminated glass web is broken. Experiments proved this fact as well as even the beam with both panes broken can carry some load and stay stable in case of bending-stiff U-profile connection with acrylic adhesive, see Figs. 14 and 15.

Strain gauges were used to measure the stresses in important point to study the real stress distribution along the beam length and cross-section, see Fig. 16.

These gauges were placed on both sides of the glass panels and measured the stress even after the breakage of one layer of double-layered glass web.

\section{Modification of Analytic Solution}

An analytic Möhler's method for hand calculation of bended elements with semi-rigid shear connection was applied to describe the stress distribution along the cross-section of hybrid beam, see Chapter 5. Different types of connection details and used kind of adhesive lead to different stress distribution in cross section, see Fig. 17.
Main task of the modification was to include the change-ability of adhesive shear modulus during the range of load, which significantly affects the results. For example, the real shear stiffness (modulus) of acrylic adhesive decreases by increasing shear load, see also the results of small-scale connection tests in Fig. 5. Main fault, caused by taking only the initial value of the shear modulus of adhesive into account, is

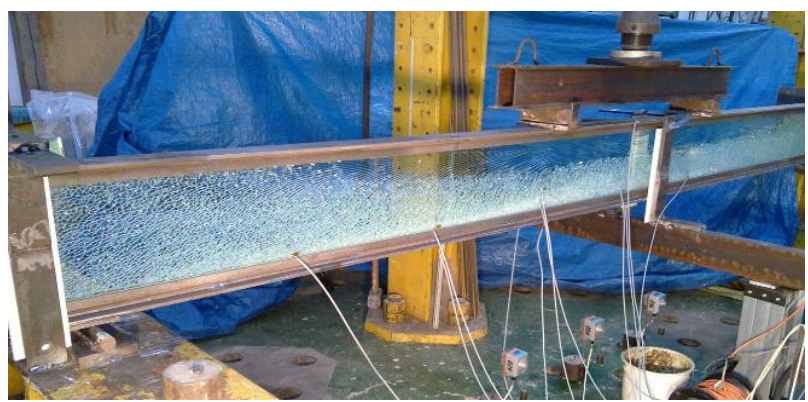

Fig. 14 Hybrid beam during the experiment with one broken pane (double layered web).

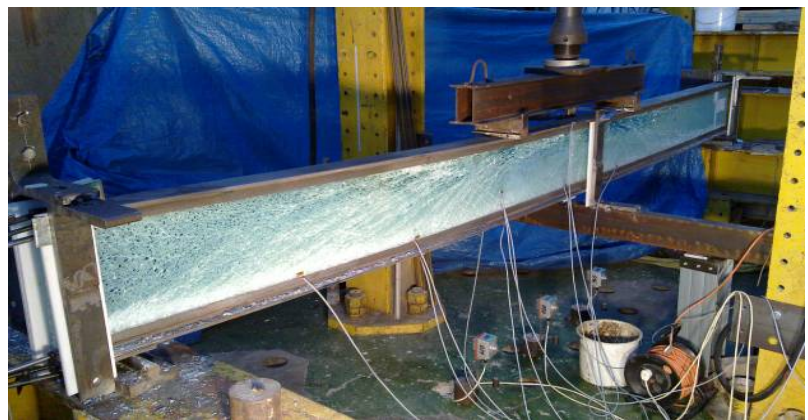

Fig. 15 Hybrid beam with both panes broken.

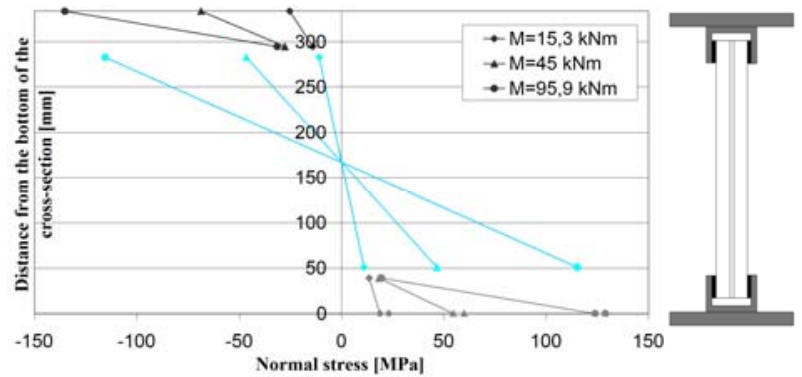

Fig. 16 Stress distribution along the cross section with U-profile connection and acrylic adhesive.

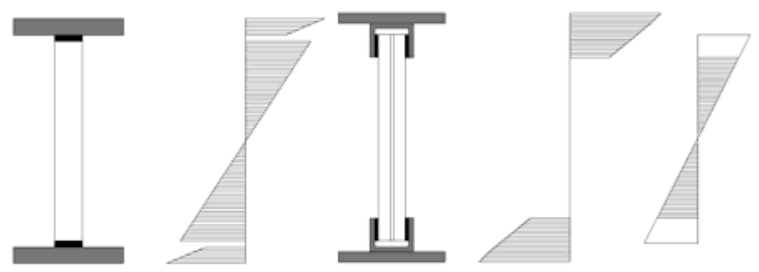

Fig. 17 Stress distribution along different cross sections. 
assumption of the higher degree of shear interaction between steel and glass than the current value at specific state of stress of the adhesive layer. It leads to unsafe design of whole beam, because of the consideration of lower normal stresses on the edge of the glass pane. True stresses caused by the same bending moment are higher because shear modulus of adhesive decreases by increasing stress and whole joint loses its stiffness, see Fig. 18, determined for direct connection and acrylic adhesive.

Applied Möhler's method was therefore modified by author in accordance with experimentally proved results. Influence of the decreasing shear modulus of adhesive in connection on increasing normal stress on the edge of the glass pane was determined for each adhesive and type of connection. Separately defined secant shear modulus of adhesive in corresponding values of normal stress in the glass web (as measured quantity) were find to reach the agreement of measured stress distribution in cross section and predicted by Möhler's method. In Fig. 19, there is a comparison of experimentally measured results and Möhler's method modified for beams with polymer adhesive with changeable shear modulus, described above. Enough accurate prediction of the stress distribution is significant.

\section{Numerical Studies}

Finite element software package Ansys was applied to create properly working non-linear material models of different adhesives in overlapped connection, subjected to shear, see chapter 4 . The best fitted models were linear elastic for stiff epoxy resin, multi-linear elastic for acrylic and hyper-elastic Mooney-Rivlin for polyurethanes and silicones. These models were later on fine-tuned according to the real thickness of the joint and included into a complex FE model of the hybrid beam, see Fig. 20.

Resulting stress distribution in the glass part of the beam, with U-profile connection and acrylic adhesive, subjected to bending moment $\mathrm{M}=60 \mathrm{kNm}$, which caused normal stress in the double layered $2 \times 12 \mathrm{~mm}$

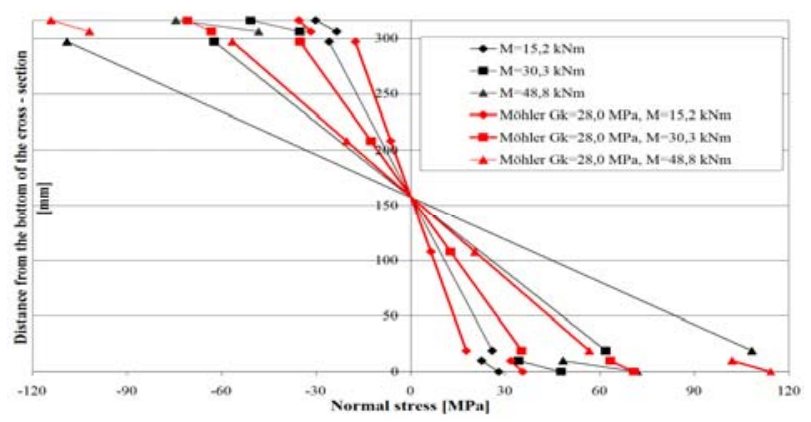

Fig. 18 Unsafe stress prediction by non-modified Möhler.

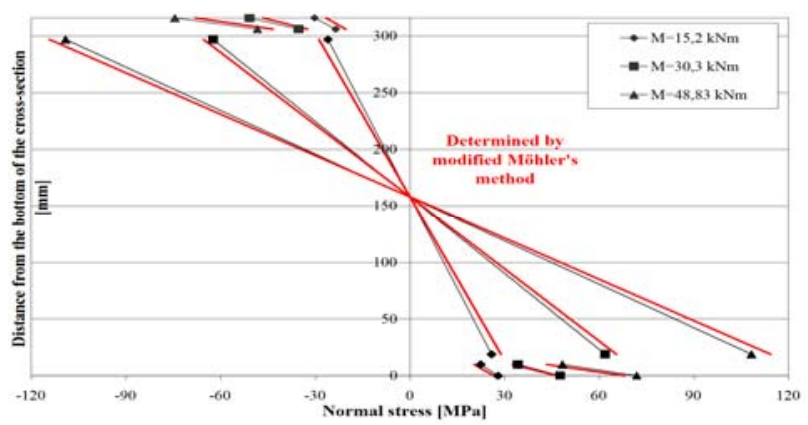

Fig. 19 Accurate stress prediction by modified Möhler.

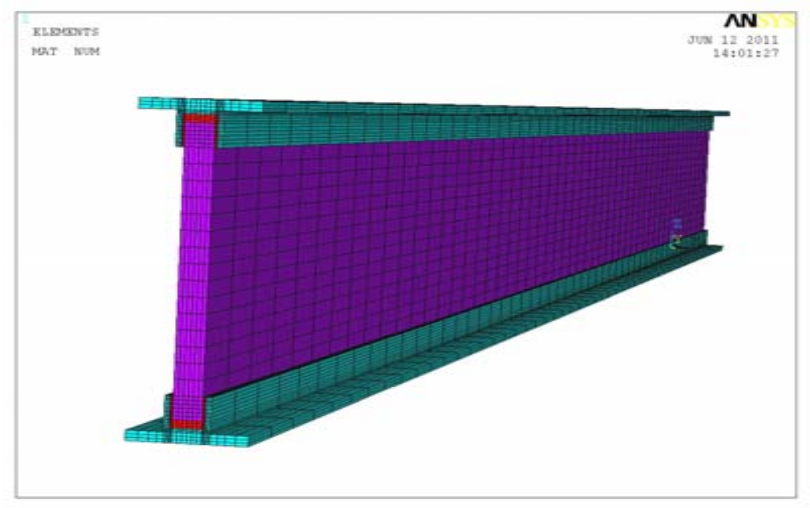

Fig. 20 FE model of the beam with U-profile connection.

toughened glass pane close to $70 \mathrm{MPa}$, is in Fig. 21 . Results of numerical study were compared with experiments with satisfying agreement, see Table 1 .

Numerical simulation of hybrid beam under increasing load and bending moment led to accurate prediction of the behavior of the hybrid beam as well as determining of important values of stresses and deflections, which were slightly higher than experimentally obtained, therefore can be assumed on the safe side. Numerical approach confirmed, that FE analysis is a strong tool for designing or to prove existing structure. 


\section{Design Guidance}

Design graphs and tables were created based on the analytical method, proved by experiments and supported by properly working numerical studies. Table 2 was created for direct connection between web and flanges and acrylic adhesive assuming the design tension strength of toughened glass $\mathrm{f}_{\mathrm{td}}=80 \mathrm{MPa}$. First proposal of the glass pane height can be found there if steel flanges are assumed as a $100 \times 10 \mathrm{~mm}$ cross section of steel grade S235. Correction factors of the values in the table came out of parametric studies when flanges are different. Grey hatched fields in the table mean values, where design shear strength of the adhesive determined the load bearing capacity of the beam (not tension strength of the glass). The same values of minimum web heights for different loadings mean, that vertical deflection caused by proper service load was resulting.

Value obtained by the designer from such a table is only the first proposal adequate evaluation always has to be performed according to specific load conditions and robustness requirements, which are not included.

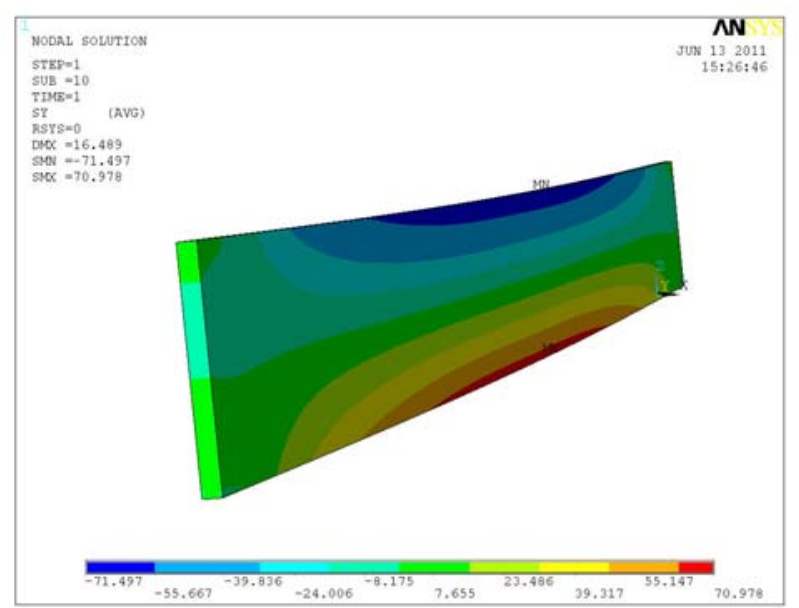

Fig. 21 Results of FE model of the beam with U-profile connection.

Table 1 Values of stress and deflection of the hybrid beam under bending moment $60 \mathrm{kNm}$, comparison of numerical studies and experiments.

\begin{tabular}{lll}
\hline Value & Exp. & Num. \\
\hline Stress in the glass (MPa) & 69.3 & 71.0 \\
Vertical deflection (mm) & 15,0 & 16,6 \\
\hline
\end{tabular}

Table 2 Design table for estimation of glass web height and thickness for the beam with direct connection and acrylic adhesive.

\begin{tabular}{|c|c|c|c|c|c|c|c|c|c|c|c|c|}
\hline \multirow{4}{*}{$\begin{array}{l}\text { Ultimate } \\
\text { design load } \\
(\mathrm{kN} / \mathrm{m})\end{array}$} & \multicolumn{12}{|c|}{ Span of the beam } \\
\hline & \multicolumn{3}{|c|}{$2 \mathrm{~m}$} & \multicolumn{3}{|c|}{$4 \mathrm{~m}$} & \multicolumn{3}{|c|}{$6 \mathrm{~m}$} & \multicolumn{3}{|c|}{$8 \mathrm{~m}$} \\
\hline & \multicolumn{3}{|c|}{ Thickness of the glass web } & \multicolumn{3}{|c|}{ Thickness of the glass web } & \multicolumn{3}{|c|}{ Thickness of the glass web } & \multicolumn{3}{|c|}{ Thickness of the glass web } \\
\hline & $10 \mathrm{~mm}$ & $20 \mathrm{~mm}$ & $30 \mathrm{~mm}$ & $10 \mathrm{~mm}$ & $20 \mathrm{~mm}$ & $30 \mathrm{~mm}$ & $10 \mathrm{~mm}$ & $20 \mathrm{~mm}$ & $30 \mathrm{~mm}$ & $10 \mathrm{~mm}$ & $20 \mathrm{~mm}$ & $30 \mathrm{~mm}$ \\
\hline 0.5 & 100 & 100 & 100 & 200 & 200 & 200 & 200 & 250 & 250 & 200 & 300 & 350 \\
\hline 1 & 100 & 100 & 100 & 200 & 200 & 200 & 200 & 250 & 250 & 200 & 300 & 350 \\
\hline 2 & 100 & 100 & 100 & 200 & 200 & 200 & 200 & 250 & 250 & 240 & 300 & 350 \\
\hline 3 & 100 & 100 & 100 & 200 & 200 & 200 & 230 & 250 & 250 & 320 & 300 & 350 \\
\hline 4 & 100 & 100 & 100 & 200 & 200 & 200 & 280 & 250 & 250 & 395 & 300 & 350 \\
\hline 5 & 115 & 100 & 100 & 200 & 200 & 200 & 325 & 250 & 250 & 460 & 300 & 350 \\
\hline 6 & 125 & 100 & 100 & 220 & 200 & 200 & 370 & 250 & 250 & 520 & 300 & 350 \\
\hline 8 & 145 & 100 & 100 & 265 & 200 & 200 & 445 & 255 & 250 & 620 & 345 & 350 \\
\hline 10 & 170 & 115 & 100 & 310 & 200 & 200 & 510 & 295 & 250 & 720 & 405 & 350 \\
\hline 12 & 190 & 125 & 100 & 345 & 225 & 200 & 565 & 335 & 260 & 820 & 455 & 350 \\
\hline 14 & 205 & 135 & 110 & 380 & 250 & 200 & 620 & 370 & 285 & 920 & 505 & 385 \\
\hline
\end{tabular}

\section{Conclusions}

A wide range of adhesives with a diverse spread of stiffness, strength and ductility properties, consisting of silicone, polyurethane, acrylic, and epoxy resin were considered for potential use in hybrid steel-glass beams. Comprehensive physical testing was performed and material properties of adhesives were obtained. The shear connection tests served to determine the shear stress-sliding behavior of the joints. In addition, 
the influence of thickness on the load bearing capacity was determined. Based on the physical testing and the finite element studies analyzing the behavior of the adhesive under increasing load, material laws were developed for each of the tested adhesives.

An extensive set of large scale tests were performed on hybrid steel-glass beams using the adhesives proposed from the adhesive connection tests and different details of connection between web and flanges. From this extensive set of full-scale tests the performance of hybrid beams under bending was determined. Finally, design guidance was presented. Further research is needed for defining safe and reliable design rules. Main topics to be investigated are long-term load behavior, ageing effects of adhesives, protection from fire and corrosion, cyclic load, lateral torsion buckling, robustness and possibilities of use of another material e.g. stainless steel.

\section{Acknowledgment}

This research, experimental evaluations and numerical investigations were supported by the grant SGS10/237/OHK1/3T/11.

\section{References}

[1] A. B. Ølgaard, J. H. Nielsen and J. H. Olesen, Design of mechanically reinforced glass beams modelling and experiments, Structural Engineering International 2 (2009) 130-136.

[2] C. Louter, Fragile Yet Ductile-Structural Aspects of Reinforced Glass Beams, Wöhrmann Print Service, Netherlands, 2011.

[3] B. Freytag, Glass-concrete composite technology, Structural Engineering International 14 (2) (2004) 111-117.

[4] B. Abeln and E. Preckwinkel, Entwicklung hybrider Stahl-Glas-Träger, Ernst\&Sohn, Stahlbau 4 (2011) 218-225.

[5] M. Netusil and M. Eliasova, Behaviour of the glued joint in hybrid steel-glass beam, Pollack Periodica-An International Journal for Engineering and Information Sciences 5 (1) (2010) 97-108.

[6] K. Möhler, Über das Tragverhalten von Biegeträgern und Druckstäben mit zusammengesetzten Querschnitten und nachgiebigen Verbindungsmitteln. TH Karlsruhe, 1956, Technischen Hochschule Fridericiana zu Karlsruhe. 